\title{
Towards the Development of an Interval Arithmetic Environment for Validated Computer-Aided Design and Verification of Systems in Control Engineering
}

\author{
Andreas Rauh, Johanna Minisini, and Eberhard P. Hofer \\ Institute of Measurement, Control, and Microtechnology \\ University of Ulm \\ D-89069 Ulm, Germany \\ \{Andreas.Rauh, Johanna.Minisini, Eberhard.Hofer\} @uni-ulm.de
}

\begin{abstract}
In this paper, an overview of the potential use of validated techniques for the analysis and design of controllers for linear and nonlinear dynamical systems with uncertainties is given. In addition to robust pole assignment for linear dynamical systems with parameter uncertainties, mathematical system models and computational techniques are considered in which constraints for both state and control variables are taken into account. For that purpose, the use of interval arithmetic routines for calculation of guaranteed enclosures of the solutions of sets of ordinary differential equations and for the calculation of validated sensitivity measures of state variables with respect to parameter variations are discussed. Simulation results as well as further steps towards the development of a general-purpose interval arithmetic framework for the design and verification of systems in control engineering are summarized.
\end{abstract}

\section{Introduction}

Modern techniques for the design and analysis of control strategies for nonlinear dynamical systems are often based on the simulation of the open-loop as well as the closed-loop behavior of suitable mathematical models described by continuous-time and discrete-time state-space representations. In addition to sets of ordinary differential equations (ODEs) and difference equations, sets of differential algebraic equations (DAEs) are commonly used in control engineering. Since we will focus on computational techniques which are applicable to the design and mathematical verification of controllers for lumped parameter systems, i.e., systems which do not contain elements with distributed parameters, partial differential equations will not be considered in this paper.

The prerequisite for the design and robustness analysis of each control system is the identification of mathematical models which describe the dynamics of the plant to be controlled as well as the available measurement devices with a sufficient accuracy. The model identification task comprises the derivation of physically motivated state equations, their parameterization based on measured data, the identification of uncertainties, as well as simplifications to apply specific approaches for controller design.

Since dynamical system models are subject to uncertain parameters and uncertain initial conditions in most practical applications, detailed mathematical specifications 
of the desired dynamics of the controlled system are necessary. These involve the definition of robustness with respect to uncertainties. For linear system representations, robustness is commonly specified in terms of regions in the complex domain containing all admissible poles of the closed-loop transfer functions ( $\Gamma$-stability) or in terms of specifications of worst-case bounds for the frequency response ( $\mathscr{B}$-stability) $[1,10]$.

However, these specifications do not allow for inclusion of state constraints in the time-domain which are often available if controllers are designed for safety-critical applications. In general, pole assignment after linearization of the state equations is not sufficient for nonlinear systems since the asymptotic stability of the resulting closedloop dynamics has to be proven regardless which eigenvalues are chosen.

In Section 2, an interval arithmetic framework for the design of robust controllers for linear systems with parameter uncertainties is introduced. This approach provides a guaranteed solution for $\Gamma$-stability-based robust controller design by calculating both inner and outer enclosures of the admissible parameters of controllers with a predefined structure. Using the time-domain approach summarized in Section 3, constraints for both the state and control vectors can be mapped into the parameter space. In contrast to the $\Gamma$-stability approach, it is directly applicable to nonlinear systems. Possible combinations with routines for robust pole assignment and optimal control are highlighted. In Section 4, an extension of the validated initial value problem solver VALENCIAIVP is introduced to compute differential sensitivities of the trajectories of all state variables with respect to variations of system parameters as well as the adaptation of controller parameters. This approach is used to analyze a feedforward control strategy for a simplified model of biological wastewater treatment plants in Section 5. Finally, conclusions and an outlook on future research are given in Section 6.

\section{Robust Pole Assignment Using Interval Techniques}

In this paper, the design of closed-loop controllers for dynamical systems described by sets of ODEs

$$
\dot{x}(t)=f(x(t), p(t), u(t)) \quad \text { with } \quad x \in \mathbb{R}^{n_{x}}, p \in \mathbb{R}^{n_{p}}, u \in \mathbb{R}^{n_{u}}
$$

is discussed. First, robust pole assignment for linear dynamical systems

$$
\dot{x}(t)=A(p) \cdot x(t)+B(p) \cdot u(t)
$$

will be addressed, where the control laws under consideration are given by

$$
u(t)=-k \cdot x(t) \quad \text { with } \quad k \in \mathbb{R}^{n_{u} \times n_{x}} .
$$

It is well known that for linear, fully state-controllable systems with exactly known parameters $p$, suitable feedback gain matrices $k$ can be determined such that the closedloop system has any desired eigenvalues. For single-input-single-output systems, a closed-form expression for pole assignment has been derived by Ackermann [1]. However, for systems with multiple control inputs, this problem is underdetermined such that additional assumptions (which are related to certain robustness or optimality criteria as well as decoupling properties) are necessary to find a unique parameterization of the control law (3). 
For linear systems with bounded uncertainties of the parameters $p$, pole assignment can be generalized to the assignment of domains of admissible eigenvalues. In control engineering, such specifications are usually referred to as $\Gamma$-stability domains. Let the characteristic polynomial of the closed-loop control system be defined by

$$
a(s, p, k):=\operatorname{det}\left(s \cdot I-A_{R}(p, k)\right) \quad \text { with } \quad A_{R}(p, k):=A(p)-B(p) \cdot k .
$$

The roots of the family of polynomials

$$
A(s, p, k):=\{a(s, p, k) \mid p \in[p], k \in[K]\}
$$

with

$$
[K]:=\left\{k_{i j} \mid k_{i j} \in\left[\underline{k}_{i j} ; \bar{k}_{i j}\right], i=1, \ldots, n_{u}, j=1, \ldots, n_{x}\right\}
$$

are denoted by

$$
\operatorname{Roots}[A(s, p, k)]:=\{v \in \mathbb{C} \mid a(v, p, k)=0, a(s, p, k) \in A(s, p, k)\} .
$$

A linear dynamical system is $\Gamma$-stable if all roots of its characteristic polynomial have strictly negative real parts and if they are completely included in a region $\Gamma \subset \mathbb{C}^{-}$for all possible parameters $p \in[p]$ and at least one $k$, i.e., if $\operatorname{Roots}[A(s, p, k)] \subseteq \Gamma \subset \mathbb{C}^{-}$ holds. Based on this definition, Ackermann and Kaesbauer developed an approach to determine the set of all controller parameters $k$ which are consistent with a prescribed region $\Gamma$, see e.g. [1]. This approach is based on mapping so-called real root boundaries, complex root boundaries, and infinite root boundaries into the parameter space, where all coefficients $a_{i}, i=0,1, \ldots, n_{x}$, of the characteristic polynomial $a(s, p, k)$ are real, non-negative, and continuously depending upon $p$ and $k$. Since this approach, as it is implemented in the MATLAB toolbox PARADISE [10], is mostly a graphical procedure which makes use of the boundary crossing theorem of Frazer and Duncan [2], due to which the roots of $a(s, p, k)$ depend continuously on continuous variations of $p$ and $k$, it is limited to a small number of controller parameters. For higher-dimensional problems, gridding of the parameter space is usually unavoidable.

To avoid this shortcoming and to make routines for robust pole assignment available for systems with coefficients $a_{i}$ which do not depend continuously upon the parameters $p$, an interval arithmetic routine has been developed to exclude intervals $[k] \subset[K]$ for the controller gains from an a-priori given enclosure $[K]$ if at least one eigenvalue is certainly not included in $\Gamma$ for at least one $p \in[p]$ and for every possible $k \in[k]$.

In Fig. 1, the interval algorithm for pole assignment which has been implemented in MATLAB using the interval arithmetic toolbox INTLAB [9] is summarized. In this algorithm, the roots of $a(s, p, k)$ are enclosed by the interval boxes $\left[\lambda_{R, i}(k)\right], i=1, \ldots, n_{x}$.

For a subdivision of $[K]$ into several subintervals $[k] \subset[K]$ obtained by application of the criteria from Fig. 1, the inner interval enclosure of the set of admissible controller gains is given by

$$
\mathscr{K}_{\Gamma, i}:=\left\{[k] \mid\left[\lambda_{R, i}(k)\right] \subseteq \Gamma, \forall p \in[p], \forall k \in[k] \subset[K], i=1, \ldots, n_{x}\right\} .
$$

Its outer enclosure, for which $\mathscr{K}_{\Gamma, i} \subseteq \mathscr{K}_{\Gamma, o}$ holds, results from eigenvalues $\left[\lambda_{R, i}(k)\right]$ which are not completely outside of $\Gamma$ according to

$$
\mathscr{K}_{\Gamma, o}:=\left\{[k] \mid\left[\lambda_{R, i}(k)\right] \cap \Gamma \neq \emptyset, \forall p \in[p], \forall k \in[k] \subset[K], i=1, \ldots, n_{x}\right\} .
$$




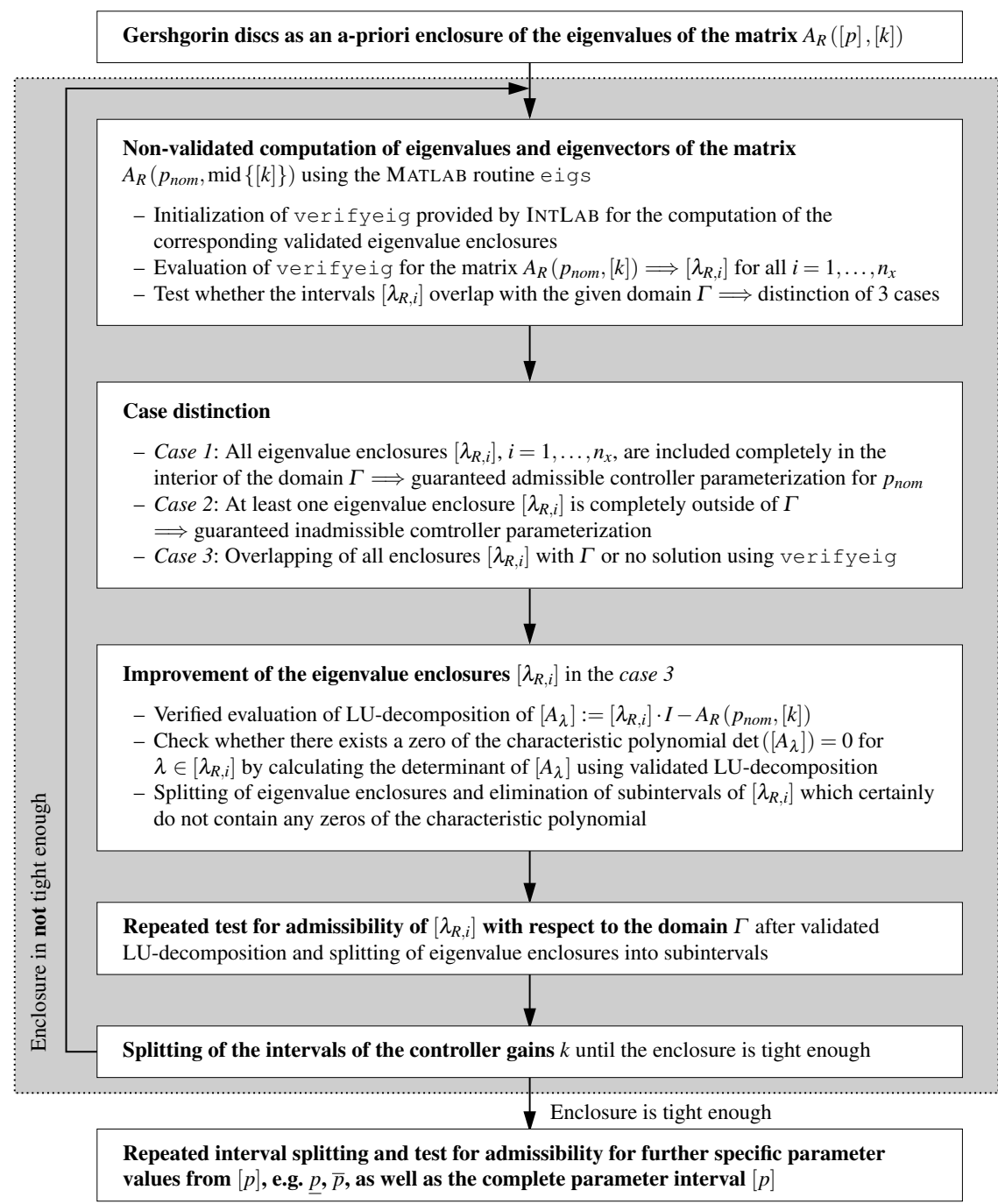

Fig. 1: Interval algorithm for robust pole assignment with given eigenvalue domains $\Gamma \subset \mathbb{C}^{-}$.

In the following, the double integrating plant

$$
\dot{x}(t)=\left[\begin{array}{cc}
0 & \alpha \\
0 & 0
\end{array}\right] x(t)+\left[\begin{array}{l}
0 \\
1
\end{array}\right] u(t) \quad \text { with } \quad \alpha \in[\alpha]:=[0.9 ; 1.1]
$$

and $u:=-k \cdot x=\left[k_{1} k_{2}\right] \cdot x, k \in[K]=[[-10 ; 10][-10 ; 10]]$ is considered as a simple application scenario to visualize the interval routine for robust pole assignment. Using this procedure, guaranteed interval enclosures of the regions of admissible controller gains have been determined for both the nominal system parameter $\alpha_{\text {nom }}=1.0$ and the 


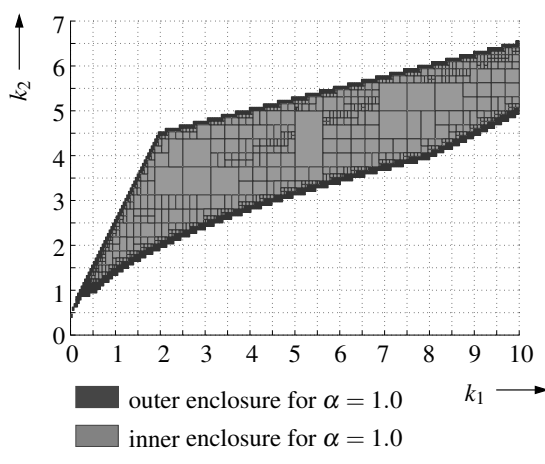

(a) Controller gains for $\alpha=\alpha_{\text {nom }}$.

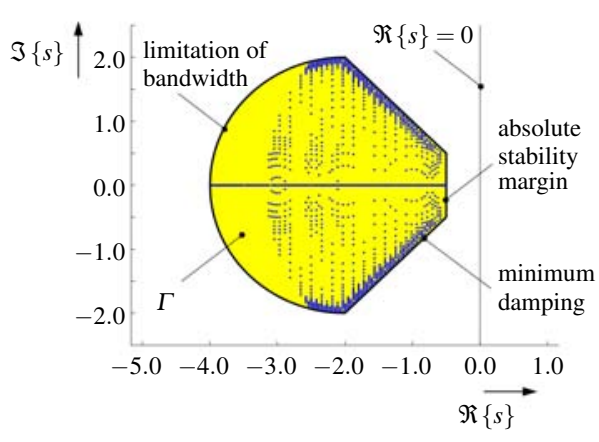

(c) Eigenvalues corresponding to the inner enclosure in Fig. 2(a), $\alpha=\alpha_{\text {nom }}$.

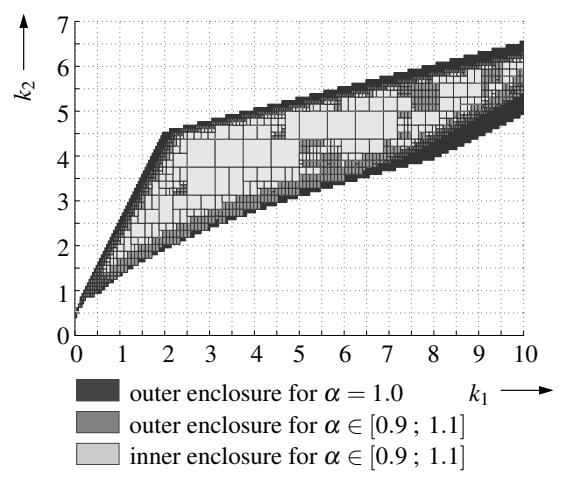

(b) Comparison of admissible controller gains for $\alpha=\alpha_{\text {nom }}$ and $\alpha \in[\alpha]$.

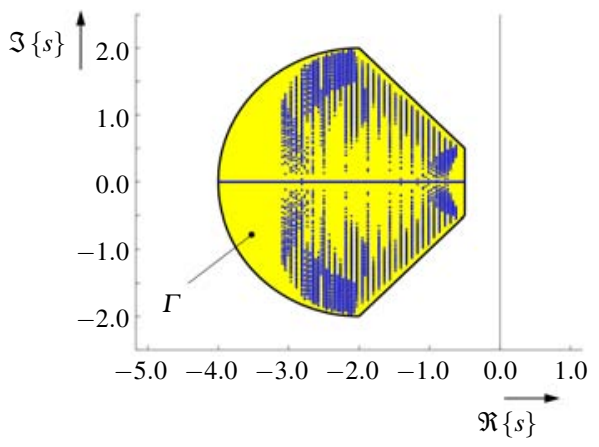

(d) Eigenvalues corresponding to the inner enclosure in Fig. 2(b), $\alpha \in[\alpha]$.

Fig. 2: Enclosures of the controller gains $k_{1}$ and $k_{2}$ which are consistent with the $\Gamma$-stability domain and visualization of the corresponding eigenvalues for $\alpha_{\text {nom }}=1.0$ and $[\alpha]=[0.9 ; 1.1]$ with a limited number of subdivisions of the a-priori enclosure $[K]$.

uncertain parameter $[\alpha]$. In Fig. 2, the inner and outer enclosures of the admissible controller gains defined in (8) and (9) are depicted together with the resulting eigenvalues for gain factors from the enclosures $\mathscr{K}_{\Gamma, i}$. The domain $\Gamma$ is defined as shown in the Figs. 2(c) and 2(d). For illustration purposes, the eigenvalues have only been computed for the vertices of the interval boxes $[k]$ of the corresponding inner enclosures.

As shown in this example, the controller parameterization is usually not unique, since arbitrary gain factors from the computed inner interval enclosures $\mathscr{K}_{\Gamma, i}$ are consistent with the robustness specifications. At the end of the following Section, possible further criteria are discussed which can be applied to obtain a unique parameterization. Furthermore, it should be pointed out that the interval arithmetic routine presented in this Section does not make any assumptions whether the eigenvalue domain $\Gamma$ consists of a single region or of the union of several disconnected domains. 


\section{Time-Domain Approach for Robust Controller Design}

In contrast to robust pole assignment, the time-domain approach presented in the following is also applicable to nonlinear systems without the necessity to linearize the state equations for parameterization of controllers with a given structure. As for the interval arithmetic approach for robust pole assignment, a-priori bounds $[K]$ for the feedback gain matrix in (3) are assumed to be given. In addition to linear controllers, any other parameterized control law can be considered. The time-domain approach relies on calculating guaranteed enclosures of the trajectories of the states of the control system

$$
\dot{x}(t)=f(x(t), p(t), u(x(t), w(t), k)) \quad \text { with } \quad p \in[p], w(t) \in[w(t)], k \in[k]
$$

over a finite time horizon $t \in\left[t_{0} ; t_{f}\right]$. In $(11), w(t)$ denotes a given reference signal. To compute guaranteed state enclosures, validated ODE solvers such as COSY VI, VALENCIA-IVP, VNODE, or VSPODE are applicable [4]. Starting from the complete a-priori enclosure $[K]$, the intervals for the controller gains are successively split into subintervals $[k]$. All subintervals which certainly lead to a violation of prescribed time-domain constraints $\mathscr{X}(t)$ according to

$$
[x(t)] \cap \mathscr{X}(t)=\emptyset \quad \text { for at least one } t \in\left[t_{0} ; t_{f}\right]
$$

are excluded. For all other subintervals $[k]$ two cases have to be distinguished. First, intervals $[k]$ belong to the desired inner enclosure of admissible controller gains if

$$
\mathscr{K}_{T, i}:=\left\{[k] \mid[x(t)] \subseteq \mathscr{X}(t) \text { for all } t \in\left[t_{0} ; t_{f}\right]\right\}
$$

holds. Second, the corresponding outer interval enclosure is defined by

$$
\mathscr{K}_{T, o}:=\left\{[k] \mid[x(t)] \cap \mathscr{X}(t) \neq \emptyset \text { for all } t \in\left[t_{0} ; t_{f}\right]\right\} .
$$

All subintervals which neither belong to the list of inadmissible intervals nor to the inner enclosure $\mathscr{K}_{T, i}$ have to be split further to assign them to one of these two lists.

Since this approach relies on pure time-domain specifications of robustness, it is applicable to arbitrary nonlinear systems which can be handled by the above-mentioned validated ODE solvers. Especially for control laws for linear systems, which can also be parameterized efficiently using the $\Gamma$-stability approach presented in Section 2 (resp. the $\mathscr{B}$-stability approach), a combination of these different types of restrictions for the gain factors is straightforward by intersecting the resulting interval enclosures $\mathscr{K}_{\Gamma}$ and $\mathscr{K}_{T}$.

As a simple demonstration example, again the double integrating plant (10) with a linear state controller $u(x(t), w(t), k)=\left[k_{1} k_{2}\right] \cdot(w(t)-x(t))$ and the reference signal

$w(t)=\left[\begin{array}{ll}1 & 0\end{array}\right]^{T}=$ const is considered. The initial conditions are $x\left(t_{0}=0\right)=\left[\begin{array}{ll}0 & 0\end{array}\right]^{T}$. In Fig. 3(a) those subintervals from the a-priori bounds $\left[K_{1}\right]:=[0 ; 5],\left[K_{2}\right]:=[0 ; 5]$ of the controller gains are depicted that are consistent with the time-domain constraints

$$
\mathscr{X}(t):=\left\{\begin{aligned}
-1.0 \cdot 10^{-5} & \leq x_{1}(t) \leq 1.2 \text { for } 0 \leq t<0.75 \\
0.5 & \leq x_{1}(t) \leq 1.2 \text { for } 0.75 \leq t<1.0 \\
0.8 & \leq x_{1}(t) \leq 1.2 \text { for } t \geq 1.0 \\
x_{2} & =\text { unbounded for } t \geq 0
\end{aligned}\right.
$$




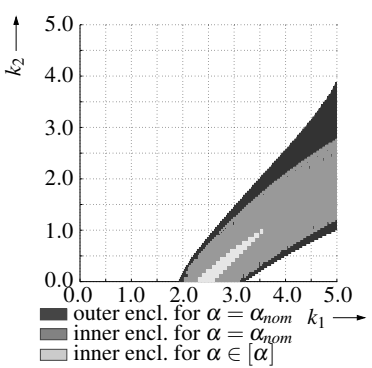

(a) Admissible gain factors for $\alpha=\alpha_{\text {nom }}$ and $\alpha \in[\alpha]$.

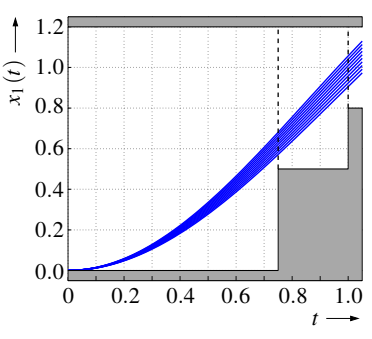

(b) Time response for $k=k^{*}$ with $\alpha \in[\alpha]$.

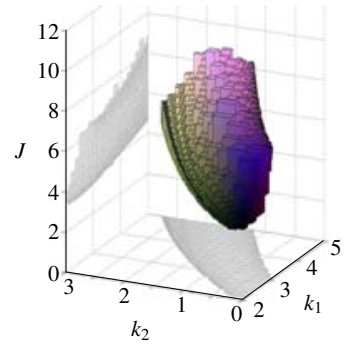

(c) Evaluation of the performance index $J$ for $\alpha=\alpha_{\text {nom }}$.

Fig. 3: Robust controller parameterization using time-domain constraints for $\left[t_{0} ; t_{f}\right]:=[0 ; 1]$.

In this example, the underlying validated evaluation of the state equations has been performed using VALENCIA-IVP for both $\alpha=\alpha_{\text {nom }}=1.0$ and $\alpha \in[\alpha]:=[0.9 ; 1.1]$. The time-domain constraints $\mathscr{X}(t)$ are guaranteed to be fulfilled for arbitrary controller gains $k$ from the inner interval enclosure $\mathscr{K}_{T, i}$. In Fig. 3(b) the time response for $x_{1}(t)$ is shown for $k=k^{*}=\left[\begin{array}{lll}2.7340 & 0.3125\end{array}\right]^{T} \in \mathscr{K}_{T, i}$ for selected $\alpha \in[\alpha]$.

Instead of choosing an arbitrary gain vector $k$ which is consistent with the constraints $\mathscr{X}(t)$, systematic approaches such as optimality criteria can be used. In the previous example, validated evaluation of the integral performance index

$$
J=\int_{t_{0}}^{t_{f}}\left(\left(x_{1}(t)-1\right)^{2}+x_{2}(t)^{2}+\left(k_{1} \cdot\left(1-x_{1}(t)\right)-k_{2} \cdot x_{2}(t)\right)^{2}\right) d t \stackrel{!}{=} \min
$$

for all $k \in \mathscr{K}_{T, i}$ using VALENCIA-IVP leads to the interval enclosures $[J]$ depicted in Fig. 3(c). The cost function (16) quantifies the deviation of the system states $x(t)$ from the desired final state $x_{1}=1$ and $x_{2}=0$ as well as the required effort for the control action over the time horizon $\left[t_{0} ; t_{f}\right]$. A general framework for interval arithmetic structure and parameter optimization for dynamical systems with both nominal and uncertain parameters has been presented by the authors in [7]. Using the definition of optimality for uncertain systems which has been introduced therein, a gain factor is optimal if it leads to the smallest upper bound of the performance index for all possible $p \in[p]$.

In addition to optimality criteria, also sensitivity measures for the system states $x(t)$ and as well as the performance index $J$ with respect to the uncertain parameters can be taken into account to find a unique robust controller parameterization. In Section 4, an interval-based approach for calculation of guaranteed enclosures of such differential sensitivity measures is introduced using an extension of VALENCIA-IVP.

\section{Validated Sensitivity Analysis Using VALENCIA-IVP}

In the following, ODEs $\dot{x}(t)=f(x(t), p)$ are considered which describe both the openloop and closed-loop system behavior, where the vector $p$ consists of all time-invariant 
system parameters as well as all controller parameters. The differential sensitivities of the solution $x(t)$ with respect to the parameters $p$ are defined by the state equations

$$
\dot{s}_{i}(t)=\frac{\partial f(x(t), p)}{\partial x} \cdot s_{i}(t)+\frac{\partial f(x(t), p)}{\partial p_{i}} \text { for all } i=1, \ldots, n_{p} .
$$

The new state vectors $s_{i}(t)$ in (17) are given by

$$
s_{i}(t):=\frac{\partial x(t)}{\partial p_{i}} \in \mathbb{R}^{n_{x}} \quad \text { with } \quad s_{i}\left(t_{0}\right)=\frac{\partial x\left(t_{0}, p\right)}{\partial p_{i}} .
$$

For initial states $x\left(t_{0}\right)$ which are independent of $p$ the equality $s_{i}\left(t_{0}\right)=0$ holds for the corresponding initial conditions of $s_{i}$. In VALENCIA-IVP, the ODEs (17) do not need to be derived symbolically, since all required partial derivatives are computed by algorithmic differentiation using FADBAD++. To obtain guaranteed enclosures of $s_{i}(t)$, the ODEs (17) are evaluated for the validated state enclosures $[x(t)]$ containing all reachable states. For both exactly known and uncertain values of the parameters $p$ and the initial states $x\left(t_{0}\right)$, the intervals $\left[s_{i}(t)\right]$ are determined such that the partial derivatives of all reachable states with respect to all possible $p_{i}$ are included. For time-varying parameters $p(t)$, the sensitivities $s_{i}(t)$ are computed with respect to time-invariant variables $\varepsilon_{i} \approx 0$ after substituting $p(t)+\varepsilon$ with $\varepsilon \in \mathbb{R}^{n_{p}}$ for the parameters $p(t)$.

\section{Sensitivity Analysis of a Wastewater Treatment Process}

The procedure for validated sensitivity analysis is demonstrated for the subsystem model of biological wastewater treatment depicted in Fig 4 which is a simplification of the Activated Sludge Model No. 1 of the International Water Association [3]. The concentration $S$ of biodegradable organic substrate is reduced by heterotrophic bacteria with the concentration $X$ under external oxygen supply with the flow rate $u_{O 2}$. The concentration of dissolved oxygen in the aeration tank is denoted by $S_{O}$. The bacteria concentration in the settler, which is modeled as a perfect separator of sludge and purified water, is denoted by $X_{S e t}$. A portion of the activated sludge is fed back into the aeration tank with the flow rate $Q_{R S}$ of return sludge. The excess sludge $Q_{E X}$ is removed from the process.

According to $[6,8]$, this process is described by the nonlinear ODEs

$$
\begin{aligned}
\dot{S} & =\frac{Q_{W}}{V_{A}}\left(S_{W}-S\right)-\hat{\mu}_{H} \frac{S}{S+K_{S}} \frac{S_{O}}{S_{O}+K_{O S}} \frac{1}{Y} X \\
\dot{X} & =-\frac{Q_{W}}{V_{A}} X+\frac{Q_{R S}}{V_{A}}\left(X_{S e t}-X\right)+\left(\hat{\mu}_{H} \frac{S}{S+K_{S}} \frac{S_{O}}{S_{O}+K_{O S}}-b\right) X \\
\dot{S}_{O} & =\frac{Q_{W}}{V_{A}}\left(S_{O W}-S_{O}\right)-\hat{\mu}_{H} \frac{S}{S+K_{S}} \frac{S_{O}}{S_{O}+K_{O S}} \frac{1-Y}{Y} X+\frac{\rho_{O 2}}{V_{A}}\left(1-\frac{S_{O}}{S_{O, \text { sat }}}\right) u_{O 2} \\
\dot{X}_{S e t} & =\frac{Q_{W}+Q_{R S}}{V_{S e t}} X-\frac{Q_{E X}+Q_{R S}}{V_{S e t}} X_{S e t} .
\end{aligned}
$$

Assuming a constant oxygen concentration $S_{O}=\hat{S}_{O}=$ const, i.e., $\dot{S}_{O}=0$, the corresponding feedforward control for the oxygen input rate $u_{O 2}$ is defined by

$$
u_{O 2}=\frac{V_{A}}{\rho_{O 2}} \frac{S_{O, \text { sat }}}{S_{O, \text { sat }}-\hat{S}_{O}}\left(\hat{\mu}_{H} \frac{S}{S+K_{S}} \frac{\hat{S}_{O}}{\hat{S}_{O}+K_{O S}} \frac{1-Y}{Y} X-\frac{Q_{W}}{V_{A}}\left(S_{O W}-\hat{S}_{O}\right)\right) \text {. }
$$




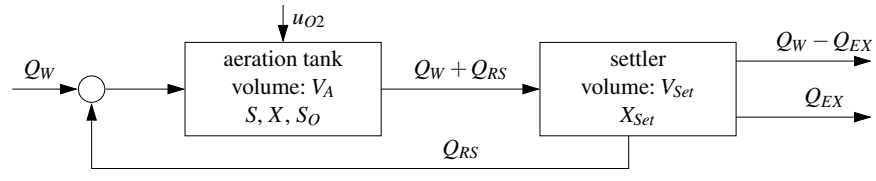

Fig. 4: Block diagram of a simplified biological wastewater treatment process.

The design and parameterization of controllers which compensate variations of states caused by parameter uncertainties relies on the adaptation of the available control variables. These are the oxygen input rate $u_{O 2}$, related to $\hat{S}_{O}$ via (20), and the flow rate of return sludge. The differential sensitivities of the state variables $S, X$, and $X_{S e t}$ w.r.t. variations $\Delta S_{O}$ and $\Delta Q_{R S}$ of the control variables and w.r.t. variations $\Delta \hat{\mu}_{H}$ with

$$
\begin{array}{ll}
S_{O}:=\hat{S}_{O} \cdot\left(1+\Delta S_{O}\right) & Q_{R S}:=Q_{R S, \text { nom }} \cdot\left(1+\Delta Q_{R S}\right) \\
\hat{\mu}_{H}:=\hat{\mu}_{H, \text { nom }} \cdot\left(1+\Delta \hat{\mu}_{H}\right) & Q_{E X}:=Q_{E X, \text { nom }}-Q_{R S} \cdot \Delta Q_{R S}
\end{array}
$$

provide the required information. For $\hat{S}_{O}=3.5 \cdot 10^{-3} \frac{\mathrm{kg}}{\mathrm{m}^{3}}$, these sensitivities have been computed using VALENCIA-IVP for three different growth rates of substrate consuming bacteria. The results of these simulations are shown in Fig. 5, where each curve represents the guaranteed enclosure for one of the considered values of $\hat{\mu}_{H}$. Note that the diameters of the resulting enclosures are below the resolution of these graphs.

According to Fig. 5, a reduced rate of the reduction of the substrate concentration $S$ caused by smaller growth rates $\hat{\mu}_{H}$ of the bacteria (leading also to a smaller concentration $X$ ) can be compensated by increasing $\hat{S}_{O}$ and/ or reducing $Q_{R S}$ to meet legal performance requirements for wastewater treatment plants which are specified e.g. in [5].

\section{Conclusions and Outlook on Future Research}

In this paper, basic interval routines for the design and analysis of controllers have been presented which are the prerequisite for nonlinear controller design. For nonlinear systems, often properties such as differential flatness or exact input-output as well as input-to-state linearizability are exploited. To generalize design procedures and to account for uncertainties and modeling errors in these cases, further interval techniques for simulation and optimization of both ODE and DAE systems will be developed in future work. VALENCIA-IVP is currently being extended to DAE systems to determine open-loop control laws matching predefined output signals in spite of uncertainties.

\section{References}

1. J. Ackermann, P. Blue, T. Bünte, L. Güvenc, D. Kaesbauer, M. Kordt, M. Muhler, and D. Odenthal. Robust Control: The Parameter Space Approach. Springer-Verlag, London, 2nd edition, 2002.

2. R. Frazer and W. Duncan. On the Criteria for the Stability of Small Motions. Proc. of the Royal Society A, 124:642-654, 1929. 


$$
\begin{aligned}
-\hat{\mu}_{H} & =0.9 \hat{\mu}_{H, n o m} \\
--\hat{\mu}_{H} & =1.0 \hat{\mu}_{H, n o m} \\
\cdots \hat{\mu}_{H} & =1.1 \hat{\mu}_{H, n o m}
\end{aligned}
$$

(a) Legend.

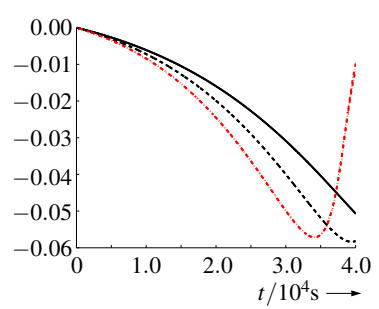

(d) Sensitivity $\frac{\partial S}{\partial \Delta S_{O}}$.

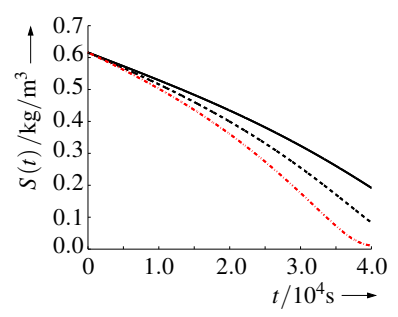

(b) Substrate concentration $S$.

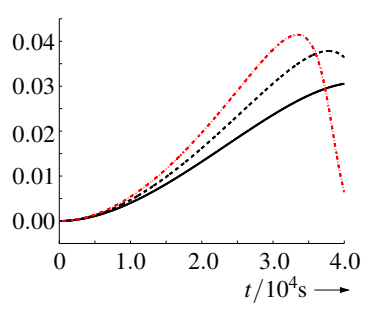

(e) Sensitivity $\frac{\partial S}{\partial \Delta Q_{R S}}$.

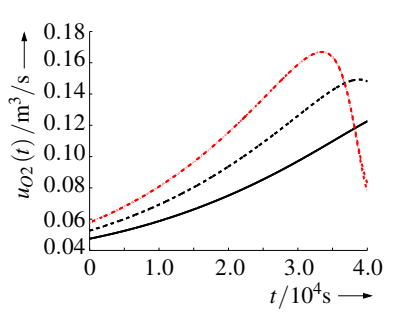

(c) Oxygen input rate.

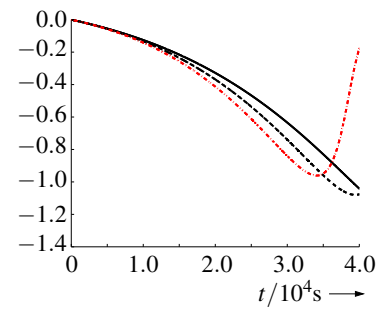

(f) Sensitivity $\frac{\partial S}{\partial \Delta \hat{\mu}_{H}}$.

Fig. 5: Sensitivity analysis of a biological wastewater treatment process for $t \in[0 ; 40,000] \mathrm{s}$.

3. M. Henze, P. Harremoës, E. Arvin, and J. la Cour Jansen. Wastewater Treatment. SpringerVerlag, Berlin, 3rd edition, 2002.

4. N. S. Nedialkov. Interval Tools for ODEs and DAEs. In CD-Proc. of the 12th GAMM-IMACS International Symposium on Scientific Computing, Computer Arithmetic, and Validated Numerics SCAN 2006, Duisburg, Germany, 2007. IEEE Computer Society.

5. Office for Official Publications of the European Communities. Council Directive of 21 May 1991 Concerning Urban Waste Water Treatment (91/271/EEC), 2003. http: // ec . europa.eu/environment/water/water-urbanwaste/directiv.html.

6. A. Rauh, E. Auer, and E. P. Hofer. VAlEnCIA-IVP: A Comparison with Other Initial Value Problem Solvers. In CD-Proc. of the 12th GAMM-IMACS International Symposium on Scientific Computing, Computer Arithmetic, and Validated Numerics SCAN 2006, Duisburg, Germany, 2007. IEEE Computer Society.

7. A. Rauh and E. P. Hofer. Interval Methods for Optimal Control. In G. Buttazzo and A. Frediani, editors, Proc. of the 47th Workshop on Variational Analysis and Aerospace Engineering, Erice, Italy, 2007. Springer-Verlag. In print.

8. A. Rauh, M. Kletting, H. Aschemann, and E. P. Hofer. Reduction of Overestimation in Interval Arithmetic Simulation of Biological Wastewater Treatment Processes. Journal of Computational and Applied Mathematics, 199(2):207-212, 2007.

9. S. M. Rump. InTLAB - INTerval LABoratory. In T. Csendes, editor, Developments in Reliable Computing, pages 77-104. Kluver Academic Publishers, 1999.

10. W. Sienel, T. Bünte, and J. Ackermann. PARADISE - PArametric Robust Analysis and Design Interactive Software Environment: A MATLAB-Based Robust Control Toolbox. In Proc. of the 1996 IEEE Intl. Symposium on Computer-Aided Control System Design, pages 380-385, Dearborn, 1996. 